hep-ph/0512249

HRI $-12 / 2005$

\title{
On Sudakov and Soft resummations in QCD
}

\author{
V. Ravindran \\ Harish-Chandra Research Institute, Chhatnag Road, Jhunsi, Allahabad, India.
}

\begin{abstract}
In this article we extract soft distribution functions for Drell-Yan and Higgs production processes using mass factorisation theorem and the perturbative results that are known upto three loop level. We find that they are maximally non-abelien. We show that these functions satisfy Sudakov type integro differential equations. The formal solutions to such equations and also to the mass factorisation kernel upto four loop level are presented. Using the soft distribution function extracted from Drell-Yan production, we show how the soft plus virtual cross section for the Higgs production can be obtained. We determine the threshold resummation exponents upto three loop using the soft distribution function.
\end{abstract}


The Drell-Yan(DY) production of di-leptons and Higgs boson production play crucial role in the hadronic colliders. The di-lepton production can not only serve as a luminosity monitor but also provide vital information on physics beyond standard model at present collider Tevatron at Fermi-Lab and future Large Hadron Collider (LHC) which is going to be up at CERN in few years. Higgs production at such colliders will establish the Standard Model(SM) as well as beyond SM Higgs [1,2]. From the theoretical side, the DY production of di-leptons and Higgs boson production are known upto Next to Next to leading order(NNLO) level in QCD. For DY at NLO level, see [3] and for the Higgs production at NLO level, see [4-6]. The NNLO contribution to DY can be found in [7-9]. Beyond NLO, the Higgs production cross sections are known only in the large top quark mass limit. For the NNLO soft plus virtual part of the Higgs production, see $[10,11]$ and the full NNLO for the Higgs production can be found in [12-14]. Apart from these fixed order results, the resummation programs for the threshold corrections to both DY and Higgs productions have also been very successful $[15,16]$. For next to next to leading logarithmic (NNLL) resummation, see $[17,18]$. Due to several important results at three loop level that are available in recent times [19]- [24], the resummation upto $N^{3} L L$ has also become reality [25-27].

With all these new results in both fixed order as well as resummed calculations, one is now able to unravel the interesting structures in the perturbative results (for example: [34-36]). Along this line, in this paper, we extract the soft distribution functions of Drell-Yan and Higgs production cross sections in perturbative QCD and show that they do not depend on the process under consideration. By that we mean that the soft distribution function of Drell-Yan production can be got entirely from the Higgs production by a simple multiplication of the colour factor $C_{F} / C_{A}$. We prove this for the pole parts upto three loop level and for the finite part we could show only to those terms that are not proportional to $\delta(1-z)$ because the three loop finite part proportional to $\delta(1-z)$ is not available yet and can be obtained only from the explicit fixed order computation of bremsstrahlung contribution. The extraction of the soft distribution function is achieved with the help of mass factorisation theorem supplemented by the recent developments in the computation of three loop anomalous dimensions, three loop form factors of quark and gluon operators and two loop bremsstrahlung contributions to Drell-Yan and Higgs productions. We discuss the consequences of our observation in the determination of soft plus virtual cross sections and the threshold resummation exponents. A brief account on the soft and jet distribution functions and the resummation exponents relevant for deep inelastic scattering (DIS) is given.

We start by writing the partonic cross section as

$$
\begin{array}{r}
\hat{\sigma}_{I}^{s v}\left(z, q^{2}, \mu_{R}^{2}\right)=\left(Z^{I}\left(\hat{a}_{s}, \mu_{R}^{2}, \mu^{2}\right)\right)^{2}\left|\hat{F}^{I}\left(\hat{a}_{s}, Q^{2}, \mu^{2}\right)\right|^{2} \delta(1-z) \otimes C e^{2 \Phi^{I}\left(\hat{a}_{s}, q^{2}, \mu^{2}, z\right)} \\
I=q, g
\end{array}
$$

with the normalisation, $\hat{\sigma}_{I, b o r n}^{s v}=\delta(1-z)$. The symbol $s v$ means that we restrict to only the soft and virtual contributions to the partonic cross sections $\hat{\sigma}_{I}^{s v}$. In the above equation we have introduced a " $C$ ordered exponential" which has the following expansion:

$$
C e^{f(z)}=\delta(1-z)+\frac{1}{1 !} f(z)+\frac{1}{2 !} f(z) \otimes f(z)+\frac{1}{3 !} f(z) \otimes f(z) \otimes f(z)+\cdots
$$


The function $f(z)$ is a distribution of the kind $\delta(1-z)$ and $\mathcal{D}_{i}$, where

$$
\mathcal{D}_{i}=\left[\frac{\ln ^{i}(1-z)}{(1-z)}\right]_{+} \quad i=0,1, \cdots
$$

and the symbol $\otimes$ means the Mellin convolution. The letters $q$ and $g$ stand for Drell-Yan(DY) and Higgs $(\mathrm{H})$ productions respectively. $q^{2}\left(=-Q^{2}\right)$ is the invariant mass of the final state (di-lepton pair in the case of DY and single Higgs boson for the Higgs production). $z$ is the scaling variable defined as the ratio of $q^{2}$ over $\hat{s}$, where $\hat{s}$ is the center of mass of the partonic system. $F^{I}\left(\hat{a}_{s}, Q^{2}, \mu^{2}\right)$ are the form factors that enter in the Drell-Yan(for $I=q$ ) and Higgs(for $I=g$ ) production cross sections. The functions $\Phi^{I}\left(\hat{a}_{s}, q^{2}, \mu^{2}, z\right)$ are called the soft distribution functions. The unrenormalised(bare) strong coupling constant $\hat{a}_{s}$ is defined as

$$
\hat{a}_{s}=\frac{\hat{g}_{s}^{2}}{16 \pi^{2}}
$$

where $\hat{g}_{s}$ is the strong coupling constant which is dimensionless in $n=4+\varepsilon$, with $n$ being the number of space time dimensions. The scale $\mu$ comes from the dimensional regularisation in order to make the bare coupling constant $\hat{g}_{s}$ dimensionless in $n$ dimensions.

The bare coupling constant $\hat{a}_{s}$ is related to renormalised one by the following relation:

$$
S_{\varepsilon} \hat{a}_{s}=Z\left(\mu_{R}^{2}\right) a_{s}\left(\mu_{R}^{2}\right)\left(\frac{\mu^{2}}{\mu_{R}^{2}}\right)^{\frac{\varepsilon}{2}}
$$

The scale $\mu_{R}$ is the renormalisation scale at which the renormalised strong coupling constant $a_{s}\left(\mu_{R}\right)$ is defined.

$$
S_{\varepsilon}=\exp \left\{\frac{\varepsilon}{2}\left[\gamma_{E}-\ln 4 \pi\right]\right\}
$$

is the spherical factor characteristic of $n$-dimensional regularisation.

The fact that $\hat{a}_{s}$ is independent of the choice of $\mu_{R}$ leads to the following renormalisation group equation (RGE) for the coupling constant:

$$
\mu_{R}^{2} \frac{d \ln a_{s}\left(\mu_{R}^{2}\right)}{d \mu_{R}^{2}}=\frac{\varepsilon}{2}+\frac{1}{a_{s}\left(\mu_{R}^{2}\right)} \beta\left(a_{s}\left(\mu_{R}^{2}\right)\right)
$$

where

$$
\beta\left(a_{s}\left(\mu_{R}^{2}\right)\right)=-a_{s}\left(\mu_{R}^{2}\right) \mu_{R}^{2} \frac{d \ln Z\left(\mu_{R}^{2}\right)}{d \mu_{R}^{2}}=-\sum_{i=0}^{\infty} a_{s}^{i+2}\left(\mu_{R}^{2}\right) \beta_{i}
$$

The solution to the above equation is given by

$$
Z\left(\mu_{R}^{2}\right)=1+a_{s}\left(\mu_{R}^{2}\right) \frac{2 \beta_{0}}{\varepsilon}+a_{s}^{2}\left(\mu_{R}^{2}\right)\left(\frac{4 \beta_{0}^{2}}{\varepsilon^{2}}+\frac{\beta_{1}}{\varepsilon}\right)+a_{s}^{3}\left(\mu_{R}^{2}\right)\left(\frac{8 \beta_{0}^{3}}{\varepsilon^{3}}+\frac{14 \beta_{0} \beta_{1}}{3 \varepsilon^{2}}+\frac{2 \beta_{2}}{3 \varepsilon}\right)
$$


The renormalisation constant $Z\left(\mu_{R}^{2}\right)$ relates the bare coupling constant $\hat{a}_{s}$ to the renormalised one $a_{s}\left(\mu_{R}^{2}\right)$ through the eqn.(5). The coefficients $\beta_{0}$ and $\beta_{1}$ are

$$
\begin{aligned}
& \beta_{0}=\frac{11}{3} C_{A}-\frac{4}{3} T_{F} n_{f} \\
& \beta_{1}=\frac{34}{3} C_{A}^{2}-4 T_{F} n_{f} C_{F}-\frac{20}{3} T_{F} n_{f} C_{A}
\end{aligned}
$$

where the color factors for $S U(N) \mathrm{QCD}$ are given by

$$
C_{A}=N, \quad C_{F}=\frac{N^{2}-1}{2 N}, \quad T_{F}=\frac{1}{2}
$$

and $n_{f}$ is the number of active flavours. In the case of the Higgs production, the number of active flavours is five because the top degrees of freedom is integrated out in the large $m_{\text {top }}$ limit.

The factors $Z^{I}\left(\hat{a}_{s}, \mu_{R}^{2}, \mu^{2}, \varepsilon\right)$ are the overall operator renormalisation constants. For the vector current $Z^{q}\left(\hat{a}_{s}, \mu_{R}^{2}, \mu^{2}\right)=1$, but the gluon operator gets overall renormalisation [28] given by

$$
\begin{aligned}
Z^{g}\left(\hat{a}_{s}, \mu_{R}^{2}, \mu^{2}, \varepsilon\right)= & 1+\hat{a}_{s}\left(\frac{\mu_{R}^{2}}{\mu^{2}}\right)^{\frac{\varepsilon}{2}} S_{\varepsilon}\left[\frac{2 \beta_{0}}{\varepsilon}\right]+\hat{a}_{s}^{2}\left(\frac{\mu_{R}^{2}}{\mu^{2}}\right)^{\varepsilon} S_{\varepsilon}^{2}\left[\frac{2 \beta_{1}}{\varepsilon}\right] \\
& +\hat{a}_{s}^{3}\left(\frac{\mu_{R}^{2}}{\mu^{2}}\right)^{3 \frac{\varepsilon}{2}} S_{\varepsilon}^{3}\left[\frac{1}{\varepsilon^{2}}\left(-2 \beta_{0} \beta_{1}\right)+\frac{2 \beta_{2}}{\varepsilon}\right]
\end{aligned}
$$

The bare form factors $\hat{F}^{I}\left(\hat{a}_{s}, Q^{2}, \mu^{2}\right)$ (before performing overall renormalisation) of both fermionic and gluonic operators satisfy the following integro differential equation that follows from the gauge as well as renormalisation group invariances [29-32]. In dimensional regularisation,

$$
Q^{2} \frac{d}{d Q^{2}} \ln \hat{F}^{I}\left(\hat{a}_{s}, Q^{2}, \mu^{2}, \varepsilon\right)=\frac{1}{2}\left[K^{I}\left(\hat{a}_{s}, \frac{\mu_{R}^{2}}{\mu^{2}}, \varepsilon\right)+G^{I}\left(\hat{a}_{s}, \frac{Q^{2}}{\mu_{R}^{2}}, \frac{\mu_{R}^{2}}{\mu^{2}}, \varepsilon\right)\right]
$$

where $K^{I}$ contains all the poles in $\varepsilon$. On the other hand, $G^{I}$ collects rest of the terms that are finite as $\varepsilon$ becomes zero. In other words $G^{I}$ contains only non-negative powers of $\varepsilon$. Since $\hat{F}^{I}$ is $\mathrm{RG}$ invariant, we find

$$
\begin{gathered}
\mu_{R}^{2} \frac{d}{d \mu_{R}^{2}} K^{I}\left(\hat{a}_{s}, \frac{\mu_{R}^{2}}{\mu^{2}}, \varepsilon\right)=-A^{I}\left(a_{s}\left(\mu_{R}^{2}\right)\right) \\
\mu_{R}^{2} \frac{d}{d \mu_{R}^{2}} G^{I}\left(\hat{a}_{s}, \frac{Q^{2}}{\mu_{R}^{2}}, \frac{\mu_{R}^{2}}{\mu^{2}}, \varepsilon\right)=A^{I}\left(a_{s}\left(\mu_{R}^{2}\right)\right)
\end{gathered}
$$

The quantities $A^{I}$ are the standard cusp anomalous dimensions and they are expanded in powers of renormalised strong coupling constant $a_{s}\left(\mu_{R}^{2}\right)$ as

$$
A^{I}\left(\mu_{R}^{2}\right)=\sum_{i=1}^{\infty} a_{s}^{i}\left(\mu_{R}^{2}\right) A_{i}^{I}
$$


The total derivative is given by

$$
\mu_{R}^{2} \frac{d}{d \mu_{R}^{2}}=\mu_{R}^{2} \frac{\partial}{\partial \mu_{R}^{2}}+\frac{d a_{s}\left(\mu_{R}^{2}\right)}{d \mu_{R}^{2}} \frac{\partial}{\partial a_{s}\left(\mu_{R}^{2}\right)}
$$

The RGE of $K^{I}$ can be solved in powers of bare coupling constant $\hat{a}_{s}$ as

$$
K^{I}\left(\hat{a}_{s}, \frac{\mu_{R}^{2}}{\mu^{2}}, \varepsilon\right)=\sum_{i=1}^{\infty} \hat{a}_{s}^{i}\left(\frac{\mu_{R}^{2}}{\mu^{2}}\right)^{i \frac{\varepsilon}{2}} S_{\varepsilon}^{i} K^{I,(i)}(\varepsilon)
$$

where,

$$
\begin{aligned}
K^{I, 1}(\varepsilon)= & \frac{1}{\varepsilon}\left(-2 A_{1}^{I}\right) \\
K^{I, 2}(\varepsilon)= & \frac{1}{\varepsilon^{2}}\left(2 \beta_{0} A_{1}^{I}\right)+\frac{1}{\varepsilon}\left(-A_{2}^{I}\right) \\
K^{I, 3}(\varepsilon)= & \frac{1}{\varepsilon^{3}}\left(-\frac{8}{3} \beta_{0}^{2} A_{1}^{I}\right)+\frac{1}{\varepsilon^{2}}\left(\frac{2}{3} \beta_{1} A_{1}^{I}+\frac{8}{3} \beta_{0} A_{2}^{I}\right)+\frac{1}{\varepsilon}\left(-\frac{2}{3} A_{3}^{I}\right) \\
K^{I, 4}(\varepsilon)= & \frac{1}{\varepsilon^{4}}\left(4 \beta_{0}^{3} A_{1}^{I}\right)+\frac{1}{\varepsilon^{3}}\left(-\frac{8}{3} \beta_{0} \beta_{1} A_{1}^{I}-6 \beta_{0}^{2} A_{2}^{I}\right) \\
& +\frac{1}{\varepsilon^{2}}\left(\frac{1}{3} \beta_{2} A_{1}^{I}+\beta_{1} A_{2}^{I}+3 \beta_{0} A_{3}^{I}\right)+\frac{1}{\varepsilon}\left(-\frac{1}{2} A_{4}^{I}\right)
\end{aligned}
$$

Similarly RGE for $G^{I}$ can also be solved and the solution is found to be

$$
\begin{aligned}
G^{I}\left(\hat{a}_{s}, \frac{Q^{2}}{\mu_{R}^{2}}, \frac{\mu_{R}^{2}}{\mu^{2}}, \varepsilon\right) & =G^{I}\left(a_{s}\left(\mu_{R}^{2}\right), \frac{Q^{2}}{\mu_{R}^{2}}, \varepsilon\right) \\
& =G^{I}\left(a_{s}\left(Q^{2}\right), 1, \varepsilon\right)+\int_{\frac{Q^{2}}{\mu_{R}^{2}}}^{1} \frac{d \lambda^{2}}{\lambda^{2}} A^{I}\left(a_{s}\left(\lambda^{2} \mu_{R}^{2}\right)\right)
\end{aligned}
$$

The integral in the above equation can be performed and it is found to be

$$
\int_{\frac{Q^{2}}{\mu_{R}^{2}}}^{1} \frac{d \lambda^{2}}{\lambda^{2}} A^{I}\left(a_{s}\left(\lambda^{2} \mu_{R}^{2}\right)\right)=\sum_{i=1}^{\infty} \hat{a}_{s}^{i}\left(\frac{\mu_{R}^{2}}{\mu^{2}}\right)^{i \frac{\varepsilon}{2}}\left[\left(\frac{Q^{2}}{\mu_{R}^{2}}\right)^{i \frac{\varepsilon}{2}}-1\right] S_{\varepsilon}^{i} K^{I,(i)}(\varepsilon)
$$

The finite function $G^{I}\left(a_{s}\left(Q^{2}\right), 1, \varepsilon\right)$ can also be expanded in powers of $a_{s}\left(Q^{2}\right)$ as

$$
G^{I}\left(a_{s}\left(Q^{2}\right), 1, \varepsilon\right)=\sum_{i=1}^{\infty} a_{s}^{i}\left(Q^{2}\right) G_{i}^{I}(\varepsilon)
$$


After substituting these solutions in the eqn.(13) and performing the final integration, we obtain the following solution

$$
\ln \hat{F}^{I}\left(\hat{a}_{s}, Q^{2}, \mu^{2}, \varepsilon\right)=\sum_{i=1}^{\infty} \hat{a}_{s}^{i}\left(\frac{Q^{2}}{\mu^{2}}\right)^{i \frac{\varepsilon}{2}} S_{\varepsilon}^{i} \hat{\mathcal{L}}_{F}^{I,(i)}(\varepsilon)
$$

where

$$
\begin{aligned}
\hat{\mathcal{L}}_{F}^{I,(1)=} & \frac{1}{\varepsilon^{2}}\left(-2 A_{1}^{I}\right)+\frac{1}{\varepsilon}\left(G_{1}^{I}(\varepsilon)\right) \\
\hat{\mathcal{L}}_{F}^{I,(2)=} & \frac{1}{\varepsilon^{3}}\left(\beta_{0} A_{1}^{I}\right)+\frac{1}{\varepsilon^{2}}\left(-\frac{1}{2} A_{2}^{I}-\beta_{0} G_{1}^{I}(\varepsilon)\right)+\frac{1}{2 \varepsilon} G_{2}^{I}(\varepsilon) \\
\hat{\mathcal{L}}_{F}^{I,(3)=} & \frac{1}{\varepsilon^{4}}\left(-\frac{8}{9} \beta_{0}^{2} A_{1}^{I}\right)+\frac{1}{\varepsilon^{3}}\left(\frac{2}{9} \beta_{1} A_{1}^{I}+\frac{8}{9} \beta_{0} A_{2}^{I}+\frac{4}{3} \beta_{0}^{2} G_{1}^{I}(\varepsilon)\right) \\
& +\frac{1}{\varepsilon^{2}}\left(-\frac{2}{9} A_{3}^{I}-\frac{1}{3} \beta_{1} G_{1}^{I}(\varepsilon)-\frac{4}{3} \beta_{0} G_{2}^{I}(\varepsilon)\right)+\frac{1}{\varepsilon}\left(\frac{1}{3} G_{3}^{I}(\varepsilon)\right) \\
\hat{\mathcal{L}}_{F}^{I,(4)=} & \frac{1}{\varepsilon^{5}}\left(\beta_{0}^{3} A_{1}^{I}\right)+\frac{1}{\varepsilon^{4}}\left(-\frac{2}{3} \beta_{0} \beta_{1} A_{1}^{I}-\frac{3}{2} \beta_{0}^{2} A_{2}^{I}-2 \beta_{0}^{3} G_{1}^{I}(\varepsilon)\right) \\
& +\frac{1}{\varepsilon^{3}}\left(\frac{1}{12} \beta_{2} A_{1}^{I}+\frac{1}{4} \beta_{1} A_{2}^{I}+\frac{3}{4} \beta_{0} A_{3}^{I}+\frac{4}{3} \beta_{0} \beta_{1} G_{1}^{I}(\varepsilon)+3 \beta_{0}^{2} G_{2}^{I}(\varepsilon)\right) \\
& +\frac{1}{\varepsilon^{2}}\left(-\frac{1}{8} A_{4}^{I}-\frac{1}{6} \beta_{2} G_{1}^{I}(\varepsilon)-\frac{1}{2} \beta_{1} G_{2}^{I}(\varepsilon)-\frac{3}{2} \beta_{0} G_{3}^{I}(\varepsilon)\right)+\frac{1}{\varepsilon}\left(\frac{1}{4} G_{4}^{I}(\varepsilon)\right)
\end{aligned}
$$

The above result is in agreement with [21], which was evaluated using various algorithms designed for solving nested sums. The cusp anomalous dimensions $A_{i}^{I}$ and $G_{i}^{I}(\varepsilon)$ are known upto order $a_{s}^{3}$. The cusp anomalous dimensions are maximally non-abelien and hence satisfy the following relation:

$$
A^{q}=\frac{C_{F}}{C_{A}} A^{g}
$$

The coefficients $G_{i}^{I}(\varepsilon)$ can be found for both $I=q$ and $I=g$ in [22] to the required accuracy in $\varepsilon$. They satisfy

$$
\begin{aligned}
& G_{1}^{I}(\varepsilon)=2\left(B_{1}^{I}-\delta_{I, g} \beta_{0}\right)+f_{1}^{I}+\sum_{k=1}^{\infty} \varepsilon^{k} g_{1}^{I, k} \\
& G_{2}^{I}(\varepsilon)=2\left(B_{2}^{I}-2 \delta_{I, g} \beta_{1}\right)+f_{2}^{I}-2 \beta_{0} g_{1}^{I, 1}+\sum_{k=1}^{\infty} \varepsilon^{k} g_{2}^{I, k}
\end{aligned}
$$




$$
\begin{aligned}
G_{3}^{I}(\varepsilon)= & 2\left(B_{3}^{I}-3 \delta_{I, g} \beta_{2}\right)+f_{3}^{I}-2 \beta_{1} g_{1}^{I, 1}-2 \beta_{0}\left(g_{2}^{I, 1}+2 \beta_{0} g_{1}^{I, 2}\right) \\
& +\sum_{k=1}^{\infty} \varepsilon^{k} g_{3}^{I, k}
\end{aligned}
$$

The constants $B_{i}^{I}$ are also known upto order $a_{s}^{3}$ thanks to the recent computation of three loop anomalous dimensions/splitting functions $[19,20]$.

The constants $f_{i}^{I}$ are analogous to the cusp anomalous dimensions $A_{i}^{I}$ that enter the form factors. It was first noticed in [33] that the single pole (in $\varepsilon$ ) of the logarithm of form factors upto two loop level $\left(a_{s}^{2}\right)$ can be predicted due the presence of these constants $f_{i}^{I}$ because they are found to be maximally non-abelien obeying the relation

$$
f_{i}^{q}=\frac{C_{F}}{C_{A}} f_{i}^{g}
$$

similar to $A_{i}^{I}$. In [22], this relation has been found to hold even at the three loop level.

The partonic cross sections $\hat{\sigma}_{I}^{s v}\left(z, q^{2}, \mu_{R}^{2}\right)$ is UV finite after the coupling constant and overall operator renormalisations are performed using $Z\left(\mu_{R}^{2}\right)$ and $Z^{I}\left(\mu_{R}^{2}\right)$. But they still require mass factorisation in order to remove the collinear divergences:

$$
\hat{\sigma}_{I}^{s v}\left(z, q^{2}, \mu_{R}^{2}, \varepsilon\right)=\Gamma^{T}\left(z, \mu_{F}^{2}, \varepsilon\right) \otimes \Delta_{I}^{s v}\left(z, q^{2}, \mu_{R}^{2}, \mu_{F}^{2}\right) \otimes \Gamma\left(z, \mu_{F}^{2}, \varepsilon\right)
$$

with $\mu_{F}$ being the factorisation scale. The resulting coefficient functions $\Delta_{I}^{s v}\left(z, q^{2}, \mu_{R}^{2}, \mu_{F}^{2}\right)$ are finite and free of collinear singularities.

$$
\Delta_{I}^{s v}\left(z, q^{2}, \mu_{R}^{2}, \mu_{F}^{2}\right)=\delta(1-z)+\sum_{i=1}^{\infty} a_{s}^{i}\left(\mu_{R}^{2}\right) \Delta_{I}^{s v,(i)}\left(z, q^{2}, \mu_{R}^{2}, \mu_{F}^{2}\right)
$$

The coefficient functions $\Delta_{I}^{s v,(i)}$ for $i=1,2$ are known(see [4] to [14]). The partial result for $\Delta_{I}^{s v,(3)}$ (i.e., all $\mathcal{D}_{i}$ except $\delta(1-z)$ are known for $i=3$ ) is also available(see [25]).

The kernel $\Gamma\left(z, \mu_{F}^{2}, \varepsilon\right)$ satisfies the following renormalisation group equation:

$$
\mu_{F}^{2} \frac{d}{d \mu_{F}^{2}} \Gamma\left(z, \mu_{F}^{2}, \varepsilon\right)=\frac{1}{2} P\left(z, \mu_{F}^{2}\right) \otimes \Gamma\left(z, \mu_{F}^{2}, \varepsilon\right)
$$

The $P\left(z, \mu_{F}^{2}\right)$ are well known Altarelli-Parisi splitting functions(matrix valued) known upto three loop level $[19,20]$ :

$$
P\left(z, \mu_{F}^{2}\right)=\sum_{i=1}^{\infty} a_{s}^{i}\left(\mu_{F}^{2}\right) P^{(i-1)}(z)
$$

The diagonal terms of splitting functions $P^{(i)}(z)$ have the following structure

$$
P_{I I}^{(i)}(z)=2\left[B_{i+1}^{I} \delta(1-z)+A_{i+1}^{I} \mathcal{D}_{0}\right]+P_{r e g, I I}^{(i)}(z)
$$


where $P_{\text {reg,II }}^{(i)}$ are regular when the argument takes the kinematic limit(here $\left.z \rightarrow 1\right)$. The RGE of the kernel can be solved in dimensional regularisation in powers of strong coupling constant. Since we are interested only in the soft plus virtual part of the cross section, only the diagonal parts of the kernels contribute. In the $\overline{M S}$ scheme, the kernel contains only poles in $\varepsilon$. Expanding the kernel in powers of bare coupling $\hat{a}_{s}$,

$$
\Gamma\left(z, \mu_{F}^{2}, \varepsilon\right)=\delta(1-z)+\sum_{i=1}^{\infty} \hat{a}_{s}^{i}\left(\frac{\mu_{F}^{2}}{\mu^{2}}\right)^{i \frac{\varepsilon}{2}} S_{\varepsilon}^{i} \Gamma^{(i)}(z, \varepsilon)
$$

we can solve the RGE for the kernel. The solutions in the $\overline{M S}$ scheme are given by

$$
\begin{aligned}
& \Gamma_{I I}^{(1)}(z, \varepsilon)=\frac{1}{\varepsilon} P_{I I}^{(0)}(z) \\
& \Gamma_{I I}^{(2)}(z, \varepsilon)=\frac{1}{\varepsilon^{2}}\left(\frac{1}{2} P_{I I}^{(0)}(z) \otimes P_{I I}^{(0)}(z)-\beta_{0} P_{I I}^{(0)}(z)\right)+\frac{1}{\varepsilon}\left(\frac{1}{2} P_{I I}^{(1)}(z)\right) \\
& \Gamma_{I I}^{(3)}(z, \varepsilon)=\frac{1}{\varepsilon^{3}}\left(\frac{4}{3} \beta_{0}^{2} P_{I I}^{(0)}(z)-\beta_{0} P_{I I}^{(0)}(z) \otimes P_{I I}^{(0)}(z)\right. \\
& \left.+\frac{1}{6} P_{I I}^{(0)}(z) \otimes P_{I I}^{(0)}(z) \otimes P_{I I}^{(0)}(z)\right)+\frac{1}{\varepsilon^{2}}\left(\frac{1}{2} P_{I I}^{(0)}(z) \otimes P_{I I}^{(1)}(z)\right. \\
& \left.-\frac{1}{3} \beta_{1} P_{I I}^{(0)}(z)-\frac{4}{3} \beta_{0} P_{I I}^{(1)}(z)\right)+\frac{1}{\varepsilon}\left(\frac{1}{3} P_{I I}^{(2)}(z)\right) \\
& \Gamma_{I I}^{(4)}(z, \varepsilon)=\frac{1}{\varepsilon^{4}}\left(\frac{1}{24} P_{I I}^{(0)}(z) \otimes P_{I I}^{(0)}(z) \otimes P_{I I}^{(0)}(z) \otimes P_{I I}^{(0)}(z)\right. \\
& -\frac{1}{2} \beta_{0} P_{I I}^{(0)}(z) \otimes P_{I I}^{(0)}(z) \otimes P_{I I}^{(0)}(z)+\frac{11}{6} \beta_{0}^{2} P_{I I}^{(0)}(z) \otimes P_{I I}^{(0)}(z) \\
& \left.-2 \beta_{0}^{3} P_{I I}^{(0)}(z)\right) \\
& +\frac{1}{\varepsilon^{3}}\left(\frac{1}{4} P_{I I}^{(0)}(z) \otimes P_{I I}^{(0)}(z) \otimes P_{I I}^{(1)}(z)-\frac{1}{3} \beta_{1} P_{I I}^{(0)}(z) \otimes P_{I I}^{(0)}(z)\right. \\
& \left.-\frac{11}{6} \beta_{0} P_{I I}^{(0)}(z) P_{I I}^{(1)}(z)+\frac{4}{3} \beta_{0} \beta_{1} P_{I I}^{(0)}(z)+3 \beta_{0}^{2} P_{I I}^{(1)}(z)\right) \\
& +\frac{1}{\varepsilon^{2}}\left(\frac{1}{3} P_{I I}^{(0)}(z) \otimes P_{I I}^{(2)}(z)+\frac{1}{8} P_{I I}^{(1)}(z) \otimes P_{I I}^{(1)}(z)-\frac{1}{6} \beta_{2} P_{I I}^{(0)}(z)\right. \\
& \left.-\frac{1}{2} \beta_{1} P_{I I}^{(1)}(z)-\frac{3}{2} \beta_{0} P_{I I}^{(2)}(z)\right)+\frac{1}{\varepsilon}\left(\frac{1}{4} P_{I I}^{(3)}(z)\right)
\end{aligned}
$$


It is now straightforward to obtain the soft distribution functions $\Phi^{I}\left(\hat{a}_{s}, q^{2}, \mu^{2}, z\right)$ from the available results known upto three loop level for the form factors $\hat{F}^{I}$, the kernels $\Gamma_{I I}$ and the coefficient functions $\Delta_{I}^{s v}$ (the $\delta(1-z)$ function part of $\Delta_{I}^{s v,(3)}$ is still unknown). The fact that $\Delta_{I}^{s v}$ are finite in the limit $\varepsilon \rightarrow 0$ implies that the soft distribution functions have pole structure in $\varepsilon$ similar to that of $\hat{F}^{I}$ and $\Gamma_{I I}$. Also, $\Phi^{I}\left(\hat{a}_{s}, q^{2}, \mu^{2}, z\right)$ satisfy the renormalisation group equation:

$$
\mu_{R}^{2} \frac{d}{d \mu_{R}^{2}} \Phi^{I}\left(\hat{a}_{s}, q^{2}, \mu^{2}, z, \varepsilon\right)=0
$$

From the above observations, it is natural to expect that the soft distribution functions also satisfy Sudakov type integro differential equation that the form factors $\hat{F}^{I}\left(Q^{2}\right)$ satisfy(see eqn.(13)). Hence,

$$
q^{2} \frac{d}{d q^{2}} \Phi^{I}\left(\hat{a}_{s}, q^{2}, \mu^{2}, z, \varepsilon\right)=\frac{1}{2}\left[\bar{K}^{I}\left(\hat{a}_{s}, \frac{\mu_{R}^{2}}{\mu^{2}}, z, \varepsilon\right)+\bar{G}^{I}\left(\hat{a}_{s}, \frac{q^{2}}{\mu_{R}^{2}}, \frac{\mu_{R}^{2}}{\mu^{2}}, z, \varepsilon\right)\right]
$$

where again $\bar{K}^{I}$ contains all the singular terms and $\bar{G}^{I}$ are finite functions of $\varepsilon$. The renormalisation group invariance leads to

$$
\begin{gathered}
\mu_{R}^{2} \frac{d}{d \mu_{R}^{2}} \bar{K}^{I}\left(\hat{a}_{s}, \frac{\mu_{R}^{2}}{\mu^{2}}, z, \varepsilon\right)=-\bar{A}^{I}\left(a_{s}\left(\mu_{R}^{2}\right)\right) \delta(1-z) \\
\mu_{R}^{2} \frac{d}{d \mu_{R}^{2}} \bar{G}^{I}\left(\hat{a}_{s}, \frac{q^{2}}{\mu_{R}^{2}}, \frac{\mu_{R}^{2}}{\mu^{2}}, z, \varepsilon\right)=\bar{A}^{I}\left(a_{s}\left(\mu_{R}^{2}\right)\right) \delta(1-z)
\end{gathered}
$$

If $\Phi^{I}\left(\hat{a}_{s}, q^{2}, \mu^{2}, z, \varepsilon\right)$ have to contain the right poles to cancel the poles coming from $\hat{F}^{I}, Z^{I}$ and $\Gamma_{I I}$ in order to make $\Delta_{I}^{s v}$ finite, then $\bar{A}^{I}$ have to satisfy

$$
\bar{A}^{I}=-A^{I}
$$

The above relation along with the renormalisation group invariance implies that

$$
\begin{aligned}
\bar{G}^{I}\left(\hat{a}_{s}, \frac{q^{2}}{\mu_{R}^{2}}, \frac{\mu_{R}^{2}}{\mu^{2}}, z, \varepsilon\right) & =\bar{G}^{I}\left(a_{s}\left(\mu_{R}^{2}\right), \frac{q^{2}}{\mu_{R}^{2}}, z, \varepsilon\right) \\
& =\bar{G}^{I}\left(a_{s}\left(q^{2}\right), 1, z, \varepsilon\right)-\delta(1-z) \int_{\frac{q^{2}}{\mu_{R}^{2}}}^{1} \frac{d \lambda^{2}}{\lambda^{2}} A^{I}\left(a_{s}\left(\lambda^{2} \mu_{R}^{2}\right)\right)
\end{aligned}
$$

Now it is now straight forward to determine all $\bar{G}^{I}\left(a_{s}\left(q^{2}\right), 1, z, \varepsilon\right)$ from the available informations. The functions $\bar{G}^{I}\left(a_{s}\left(q^{2}\right), 1, z, \varepsilon\right)$ can be expanding in powers of $a_{s}\left(q^{2}\right)$ as

$$
\bar{G}^{I}\left(a_{s}\left(q^{2}\right), 1, z, \varepsilon\right)=\sum_{i=1}^{\infty} a_{s}^{i}\left(q^{2}\right) \bar{G}_{i}^{I}(z, \varepsilon)
$$


The solution to the eqn(35) can be obtained in the way we obtained $\ln \hat{F}^{I}\left(Q^{2}\right)$. Expanding the soft distribution functions in powers of bare coupling $\hat{a}_{s}$ as

$$
\Phi^{I}\left(\hat{a}_{s}, q^{2}, \mu^{2}, z, \varepsilon\right)=\sum_{i=1}^{\infty} \hat{a}_{s}^{i}\left(\frac{q^{2}}{\mu^{2}}\right)^{i \frac{\varepsilon}{2}} S_{\varepsilon}^{i} \hat{\Phi}^{I,(i)}(z, \varepsilon)
$$

we find the solution:

$$
\hat{\Phi}^{I,(i)}(z, \varepsilon)=\hat{\mathcal{L}}_{F}^{I,(i)}(\varepsilon)\left(A^{I} \rightarrow-\delta(1-z) A^{I}, G^{I}(\varepsilon) \rightarrow \bar{G}^{I}(z, \varepsilon)\right)
$$

The finite functions $\bar{G}_{i}^{I}(z, \varepsilon)$ can be obtained using the mass factorisation formula by demanding the finiteness of the coefficient functions $\Delta_{I}^{s v,(i)}$. The RG invariance of theses soft functions and the simple rescaling $q \rightarrow(1-z) q$ imply that the following expansion is also the solution to the integro differential equation:

$$
\begin{aligned}
\Phi^{I}\left(\hat{a}_{s}, q^{2}, \mu^{2}, z, \varepsilon\right) & =\Phi^{I}\left(\hat{a}_{s}, q^{2}(1-z)^{2}, \mu^{2}, \varepsilon\right) \\
& =\sum_{i=1}^{\infty} \hat{a}_{s}^{i}\left(\frac{q^{2}(1-z)^{2}}{\mu^{2}}\right)^{i \frac{\varepsilon}{2}} S_{\varepsilon}^{i}\left(\frac{i \varepsilon}{1-z}\right) \hat{\phi}^{I,(i)}(\varepsilon)
\end{aligned}
$$

where

$$
\hat{\phi}^{I,(i)}(\varepsilon)=\hat{\mathcal{L}}_{F}^{I,(i)}(\varepsilon)\left(A^{I} \rightarrow-A^{I}, G^{I}(\varepsilon) \rightarrow \bar{G}^{I}(\varepsilon)\right)
$$

The $z$ independent constants $\overline{\mathcal{G}}^{I}(\varepsilon)$ in $\hat{\phi}^{I,(i)}(\varepsilon)$ can be obtained using the form factors, mass factorisation kernels and coefficient functions $\Delta_{I}^{s v,(i-1)}$ expanded in powers of $\varepsilon$ to the desired accuracy. This is achieved by comparing the poles as well as non-pole terms in $\varepsilon$ of $\hat{\phi}^{I,(i)}(\varepsilon)$ with those coming from the form factors, overall renormalisation constants and splitting functions and the lower $\operatorname{order} \Delta_{I}^{s v,(i-1)}$. We find

$$
\begin{aligned}
& \overline{\mathcal{G}}_{1}^{I}(\varepsilon)=-f_{1}^{I}+\sum_{k=1}^{\infty} \varepsilon^{k} \overline{\mathcal{G}}_{1}^{I,(k)} \\
& \overline{\mathcal{G}}_{2}^{I}(\varepsilon)=-f_{2}^{I}-2 \beta_{0} \overline{\mathcal{G}}_{1}^{I,(1)}+\sum_{k=1}^{\infty} \varepsilon^{k} \overline{\mathcal{G}}_{2}^{I,(k)} \\
& \overline{\mathcal{G}}_{3}^{I}(\varepsilon)=-f_{3}^{I}-2 \beta_{1} \overline{\mathcal{G}}_{1}^{I,(1)}-2 \beta_{0}\left(\overline{\mathcal{G}}_{2}^{I,(1)}+2 \beta_{0} \overline{\mathcal{G}}_{1}^{I,(2)}\right)+\sum_{k=1}^{\infty} \varepsilon^{k} \overline{\mathcal{G}}_{3}^{I,(k)}
\end{aligned}
$$

with

$$
\overline{\mathcal{G}}_{1}^{I,(1)}=C_{I} \overline{\mathcal{G}}_{1}^{(1)}
$$




$$
\begin{aligned}
= & C_{I}\left(-3 \zeta_{2}\right) \\
\overline{\mathcal{G}}_{1}^{I,(2)}= & C_{I} \overline{\mathcal{G}}_{1}^{(2)} \\
= & C_{I}\left(\frac{7}{3} \zeta_{3}\right) \\
\overline{\mathcal{G}}_{2}^{I,(1)}= & C_{I} \overline{\mathcal{G}}_{2}^{(1)} \\
= & C_{I} C_{A}\left(\frac{2428}{81}-\frac{469}{9} \zeta_{2}+4 \zeta_{2}^{2}-\frac{176}{3} \zeta_{3}\right) \\
& +C_{I} n_{f}\left(-\frac{328}{81}+\frac{70}{9} \zeta_{2}+\frac{32}{3} \zeta_{3}\right)
\end{aligned}
$$

where $C_{I}=C_{F}$ for $I=q(\mathrm{DY})$ and $C_{I}=C_{A}$ for $I=g($ Higgs $)$.

Using such compensating $\hat{\phi}^{I,(i)}(\varepsilon)$ and the following expansion,

$$
\frac{1}{1-z}\left[(1-z)^{2}\right]^{i \frac{\varepsilon}{2}}=\frac{1}{i \varepsilon} \delta(1-z)+\sum_{j=0}^{\infty} \frac{(i \varepsilon)^{j}}{j !} \mathcal{D}_{j}
$$

we obtain $\bar{G}_{i}^{I}(z, \varepsilon)$ upto three loop level. We find that the finite functions $\bar{G}_{i}^{I}(z, \varepsilon)$ have the following decomposition in terms of cusp anomalous dimension $A_{i}^{I}$ and $f_{i}^{I}$ that appear in the form factors:

$$
\begin{aligned}
\bar{G}_{1}^{I}= & -f_{1}^{I} \delta(1-z)+2 A_{1}^{I} \mathcal{D}_{0}+\sum_{k=1}^{\infty} \varepsilon^{k} \bar{g}_{1}^{I, k}(z) \\
\bar{G}_{2}^{I}= & -f_{2}^{I} \delta(1-z)+2 A_{2}^{I} \mathcal{D}_{0}-2 \beta_{0} \bar{g}_{1}^{I, 1}(z)+\sum_{k=1}^{\infty} \varepsilon^{k} \bar{g}_{2}^{I, k}(z) \\
\bar{G}_{3}^{I}= & -f_{3}^{I} \delta(1-z)+2 A_{3}^{I} \mathcal{D}_{0}-2 \beta_{1} \bar{g}_{1}^{I, 1}(z)-2 \beta_{0}\left(\bar{g}_{2}^{I, 1}(z)+2 \beta_{0} \bar{g}_{1}^{I, 2}(z)\right) \\
& +\sum_{k=1}^{\infty} \varepsilon^{k} \bar{g}_{3}^{I, k}(z)
\end{aligned}
$$

where

$$
\begin{aligned}
& \bar{g}_{1}^{I, 1}=C_{I}\left(8 \mathcal{D}_{1}-3 \zeta_{2} \delta(1-z)\right) \\
& \bar{g}_{1}^{I, 2}=C_{I}\left(-3 \zeta_{2} \mathcal{D}_{0}+4 \mathcal{D}_{2}+\frac{7}{3} \zeta_{3} \delta(1-z)\right)
\end{aligned}
$$




$$
\begin{aligned}
& \bar{g}_{1}^{I, 3}=C_{I}\left(\frac{7}{3} \zeta_{3} \mathcal{D}_{0}-3 \zeta_{2} \mathcal{D}_{1}+\frac{4}{3} \mathcal{D}_{3}-\frac{3}{16} \zeta_{2}^{2} \delta(1-z)\right) \\
& \bar{g}_{2}^{I, 1}=C_{I} C_{A}\left(\left(-\frac{1616}{27}+\frac{242}{3} \zeta_{2}+56 \zeta_{3}\right) \mathcal{D}_{0}+\left(\frac{1072}{9}-32 \zeta_{2}\right) \mathcal{D}_{1}+(-88) \mathcal{D}_{2}\right. \\
& \left.+\left(\frac{2428}{81}-\frac{469}{9} \zeta_{2}+4 \zeta_{2}^{2}-\frac{176}{3} \zeta_{3}\right) \delta(1-z)\right)+C_{I} n_{f}\left(\left(\frac{224}{27}-\frac{44}{3} \zeta_{2}\right) \mathcal{D}_{0}\right. \\
& \left.\left.+\left(-\frac{160}{9}\right) \mathcal{D}_{1}+16 \mathcal{D}_{2}+\left(-\frac{328}{81}+\frac{70}{9} \zeta_{2}+\frac{32}{3} \zeta_{3}\right) \delta(1-z)\right)\right) \\
& \bar{g}_{2}^{I, 2}=C_{I} C_{A}\left(\left(\frac{4856}{81}-\frac{938}{9} \zeta_{2}+8 \zeta_{2}^{2}-\frac{1210}{9} \zeta_{3}\right) \mathcal{D}_{0}+\left(-\frac{3232}{27}+\frac{550}{3} \zeta_{2}+112 \zeta_{3}\right) \mathcal{D}_{1}\right. \\
& \left.+\left(\frac{1072}{9}-32 \zeta_{2}\right) \mathcal{D}_{2}+\left(-\frac{616}{9}\right) \mathcal{D}_{3}\right)+C_{I} n_{f}\left(\left(-\frac{656}{81}+\frac{140}{9} \zeta_{2}+\frac{220}{9} \zeta_{3}\right) \mathcal{D}_{0}\right. \\
& \left.\left.+\left(\frac{448}{27}-\frac{100}{3} \zeta_{2}\right) \mathcal{D}_{1}+\left(-\frac{160}{9}\right) \mathcal{D}_{2}+\left(\frac{112}{9}\right) \mathcal{D}_{3}\right)\right) \\
& +\delta(1-z) \delta \bar{g}_{2}^{g, 2} \\
& \bar{g}_{3}^{I, 1}=C_{I} C_{A}^{2}\left(\left(-\frac{403861}{243}-176 \zeta_{2} \zeta_{3}+\frac{71584}{27} \zeta_{2}-\frac{5368}{15} \zeta_{2}^{2}+\frac{9272}{3} \zeta_{3}-576 \zeta_{5}\right) \mathcal{D}_{0}\right. \\
& +\left(\frac{257140}{81}-\frac{28696}{9} \zeta_{2}+\frac{1056}{5} \zeta_{2}^{2}-\frac{5632}{3} \zeta_{3}\right) \mathcal{D}_{1}+\left(-\frac{68752}{27}+\frac{1760}{3} \zeta_{2}\right) \mathcal{D}_{2} \\
& \left.+\left(\frac{7744}{9}\right) \mathcal{D}_{3}\right)+C_{I} C_{A} n_{f}\left(\left(\frac{96482}{243}-\frac{2452}{3} \zeta_{2}+\frac{1264}{15} \zeta_{2}^{2}-\frac{6536}{9} \zeta_{3}\right) \mathcal{D}_{0}\right. \\
& \left.+\left(-\frac{72008}{81}+\frac{9056}{9} \zeta_{2}+\frac{448}{3} \zeta_{3}\right) \mathcal{D}_{1}+\left(\frac{22400}{27}-\frac{320}{3} \zeta_{2}\right) \mathcal{D}_{2}+\left(-\frac{2816}{9}\right) \mathcal{D}_{3}\right) \\
& +C_{I} n_{f}^{2}\left(\left(-\frac{4480}{243}+\frac{1520}{27} \zeta_{2}+\frac{416}{9} \zeta_{3}\right) \mathcal{D}_{0}+\left(\frac{4192}{81}-\frac{736}{9} \zeta_{2}\right) \mathcal{D}_{1}\right. \\
& \left.+\left(-\frac{1600}{27}\right) \mathcal{D}_{2}+\left(\frac{256}{9}\right) \mathcal{D}_{3}\right)+C_{I} C_{F} n_{f}\left(\left(\frac{1711}{9}-60 \zeta_{2}-\frac{96}{5} \zeta_{2}^{2}-\frac{304}{3} \zeta_{3}\right) \mathcal{D}_{0}\right. \\
& \left.+\left(-220+192 \zeta_{3}\right) \mathcal{D}_{1}+(64) \mathcal{D}_{2}\right)+\delta_{g_{3}^{g}}^{g, 1} \delta(1-z)
\end{aligned}
$$

In the above equation $\delta \bar{g}_{2}^{g, 2}, \delta \bar{g}_{3}^{g, 1}$ are not known because the full fixed order $N^{3} L O$ computation 
for the soft part of the cross section is not available yet.

With the available informations(ignoring $\delta \bar{g}_{2}^{g, 2}, \delta \bar{g}_{3}^{g, 1}$ ), we find that the soft distribution functions for DY and Higgs productions are maximally non-abelien:

$$
\Phi^{q}\left(\hat{a}_{s}, q^{2}, \mu^{2}, z, \varepsilon\right)=\frac{C_{F}}{C_{A}} \Phi^{g}\left(\hat{a}_{s}, q^{2}, \mu^{2}, z, \varepsilon\right)
$$

upto three loop level. At the cross section level $\left(\Delta_{I}^{S v}\right)$, this property does not show up because of the form factors which do not have this property. The overall factors $C_{F}$ and $C_{A}$ ordinate from the leading order contributions to the soft distribution functions. Hence if you factor out this colour factor ( $C_{F}$ for the DY and $C_{A}$ for the Higgs) we find that the soft distribution functions are universal.

$$
\Phi^{I}\left(\hat{a}_{s}, q^{2}, \mu^{2}, z, \varepsilon\right)=C_{I} \Phi\left(\hat{a}_{s}, q^{2}, \mu^{2}, z, \varepsilon\right)
$$

The universality of the soft distribution functions can be understood if you notice that the soft part of the cross section is always independent of the spin, colour, flavour or any other quantum numbers after factoring out the born level cross section. It depends only on the gauge interaction, here it is $S U(N)$. This universal property can be utilised to compute soft part of the any new cross section where incoming particles carry any spin,colour,flavour or other quantum numbers. For example, if we know $\Phi\left(\hat{a}_{s}, q^{2}, \mu^{2}, z, \varepsilon\right)$ extracted from the Drell-Yan production results, we can predict the $\Delta_{g}^{s v}\left(z, q^{2}, \mu_{R}^{2}, \mu_{F}^{2}\right)$ for the Higgs production using mass factorisation formula provided we know the gluon form factor $F^{g}\left(\hat{a}_{s}, Q^{2}, \mu^{2}, \varepsilon\right)$ and the overall renormalisation constant $Z^{g}\left(\hat{a}_{s}, \mu_{R}^{2}, \mu^{2}, \varepsilon\right)$.

The soft plus virtual part of the cross section $\left(\Delta_{I}^{s v}\left(z, q^{2}, \mu_{R}^{2}, \mu_{F}^{2}\right)\right)$ using mass factorisation formula is found to be

$$
\Delta_{I}^{s v}\left(z, q^{2}, \mu_{R}^{2}, \mu_{F}^{2}\right)=\left.C \exp \left(\Psi^{I}\left(z, q^{2}, \mu_{R}^{2}, \mu_{F}^{2}, \varepsilon\right)\right)\right|_{\varepsilon=0}
$$

where $\Psi^{I}\left(z, q^{2}, \mu_{R}^{2}, \mu_{F}^{2}, \varepsilon\right)$ is a finite distribution. The $\mu_{R}$ dependence comes from the coupling constant and operator renormalisation:

$$
\begin{aligned}
\Psi^{I}\left(z, q^{2}, \mu_{R}^{2}, \mu_{F}^{2}, \varepsilon\right)= & \left(\ln \left(Z^{I}\left(\hat{a}_{s}, \mu_{R}^{2}, \mu^{2}, \varepsilon\right)\right)^{2}+\ln \left|\hat{F}^{I}\left(\hat{a}_{s}, Q^{2}, \mu^{2}, \varepsilon\right)\right|^{2}\right) \delta(1-z) \\
& +2 C_{I} \Phi\left(\hat{a}_{s}, q^{2}, \mu^{2}, z, \varepsilon\right)-2 C \ln \Gamma_{I I}\left(\hat{a}_{s}, \mu^{2}, \mu_{F}^{2}, z, \varepsilon\right)
\end{aligned}
$$

In the above equation " $C \ln$ " means the "convolution ordered" logarithm. All the products of distributions in the logarithmic expansion are understood as Mellin convolutions. The distribution $\Psi^{I}\left(z, q^{2}, \mu_{R}^{2}, \mu_{F}^{2}, \varepsilon\right)$ is regular as $\varepsilon \rightarrow 0$. The soft plus virtual cross section can be obtained by expanding $\Psi^{I}\left(z, q^{2}, \mu_{R}^{2}, \mu_{F}^{2}, \varepsilon=0\right)$ as

$$
\Psi^{I}\left(z, q^{2}, \mu_{F}^{2}, \varepsilon\right)=\sum_{i=1} a_{s}^{i}\left(\mu_{F}^{2}\right) \Psi^{I,(i)}\left(z, q^{2}, \mu_{F}^{2}\right)
$$

where we have set $\mu_{R}=\mu_{F}$ and expressing $a_{s}\left(\mu_{F}^{2}\right)$ in terms of $a_{s}\left(\mu_{R}^{2}\right)$ is straightforward. We find that the cross sections $\Delta_{I}^{s v}\left(z, q^{2}, \mu_{F}^{2}\right)$ can be obtained using

$$
\Delta_{I}^{s v,(0)}\left(z, q^{2}, \mu_{F}^{2}\right)=C_{I} \delta(1-z)
$$




$$
\begin{aligned}
\Delta_{I}^{s v,(1)}\left(z, q^{2}, \mu_{F}^{2}\right)= & \Psi^{I,(1)}\left(z, q^{2}, \mu_{F}^{2}\right) \\
\Delta_{I}^{s v,(1)}\left(z, q^{2}, \mu_{F}^{2}\right)= & \Psi^{I,(2)}\left(z, q^{2}, \mu_{F}^{2}\right)+\frac{1}{2} \Psi^{I,(1)}\left(z, q^{2}, \mu_{F}^{2}\right) \otimes \Psi^{I,(1)}\left(z, q^{2}, \mu_{F}^{2}\right) \\
\Delta_{I}^{s v,(3)}\left(z, q^{2}, \mu_{F}^{2}\right)= & \Psi^{I,(3)}\left(z, q^{2}, \mu_{F}^{2}\right)+\Psi^{I,(1)}\left(z, q^{2}, \mu_{F}^{2}\right) \otimes \Psi^{I,(2)}\left(z, q^{2}, \mu_{F}^{2}\right) \\
& +\frac{1}{6} \Psi^{I,(1)}\left(z, q^{2}, \mu_{F}^{2}\right) \otimes \Psi^{I,(1)}\left(z, q^{2}, \mu_{F}^{2}\right) \otimes \Psi^{I,(1)}\left(z, q^{2}, \mu_{F}^{2}\right)
\end{aligned}
$$

where

$$
\begin{aligned}
\Psi^{I,(1)}= & \left(\left(-2 \beta_{0} \delta_{I, g}+2 B_{1}^{I}\right) \delta(1-z)+2 A_{1}^{I} \mathcal{D}_{0}\right) \ln \left(\frac{q^{2}}{\mu_{F}^{2}}\right)+\left(3 \zeta_{2} A_{1}^{I}+2 g_{1}^{I, 1}\right. \\
& \left.+2 C_{I} \overline{\mathcal{G}}_{1}^{(1)}\right) \delta(1-z)+\left(4 A_{1}^{I}\right) \mathcal{D}_{1} \\
\Psi^{I,(2)}= & {\left[6 \zeta_{2} \beta_{0} B_{1}^{I}-6 \zeta_{2} \beta_{0}^{2} \delta_{I, g}+2 \beta_{0} g_{1}^{I, 2}+g_{2}^{I, 1}+3 \zeta_{2} A_{2}^{I}+2 \beta_{0} C_{I} \overline{\mathcal{G}}_{1}^{(2)}+C_{I} \overline{\mathcal{G}}_{2}^{(1)}\right.} \\
& +\left(-4 \beta_{1} \delta_{I, g}-2 \beta_{0} g_{1}^{(1)}+2 B_{2}^{I}-3 \zeta_{2} \beta_{0} A_{1}^{I}-2 \beta_{0} C_{I} \overline{\mathcal{G}}_{1}^{(1)}\right) \ln \left(\frac{q^{2}}{\mu_{F}^{2}}\right) \\
& \left.+\left(-\beta_{0} B_{1}^{I}+\beta_{0}^{2} \delta_{I, g}\right) \ln { }^{2}\left(\frac{q^{2}}{\mu_{F}^{2}}\right)\right] \delta(1-z)+\left[-4 \beta_{0} C_{I} \overline{\mathcal{G}}_{1}^{(1)}-2 f_{2}^{I}+2 A_{2}^{I} \ln \left(\frac{q^{2}}{\mu_{F}^{2}}\right)\right. \\
& \left.-\beta_{0} A_{1}^{I} \ln ^{2}\left(\frac{q^{2}}{\mu_{F}^{2}}\right)\right] \mathcal{D}_{0}+\left[4 A_{2}^{I}-4 \beta_{0} A_{1}^{I} \ln \left(\frac{q^{2}}{\mu_{F}^{2}}\right)\right] \mathcal{D}_{1}+\left[-4 \beta_{0} A_{1}^{I}\right] \mathcal{D}_{2}
\end{aligned}
$$$$
\Psi^{I,(3)}=\left[-30 \zeta_{2} \beta_{0} \beta_{1} \delta_{I, g}+12 \zeta_{2} \beta_{0} B_{2}^{I}-12 \zeta_{2} \beta_{0}^{2} g_{1}^{I,(1)}+6 \zeta_{2} \beta_{1} B_{1}^{I}+\frac{4}{3} \beta_{0} g_{2}^{I,(2)}+\frac{8}{3} \beta_{0}^{2} g_{1}^{I,(3)}\right.
$$$$
+\frac{4}{3} \beta_{1} g_{1}^{I,(2)}+\frac{2}{3} g_{3}^{I,(1)}+3 \zeta_{2} A_{3}^{I}-3 \zeta_{2}^{2} \beta_{0}^{2} A_{1}^{I}+\frac{4}{3} \beta_{0} C_{I} \overline{\mathcal{G}}_{2}^{(2)}+\frac{8}{3} \beta_{0}^{2} C_{I} \overline{\mathcal{G}}_{1}^{(3)}
$$$$
+\frac{4}{3} \beta_{1} C_{I} \overline{\mathcal{G}}_{1}^{(2)}+\frac{2}{3} C_{I} \overline{\mathcal{G}}_{3}^{(1)}+6 \zeta_{2} \beta_{0} f_{2}^{I}+\left(12 \zeta_{2} \beta_{0}^{3} \delta_{I, g}-6 \beta_{2} \delta_{I, g}+2 B_{3}^{I}-12 \zeta_{2} \beta_{0}^{2} B_{1}^{I}\right.
$$$$
-2 \beta_{0} g_{2}^{I,(1)}-4 \beta_{0}^{2} g_{1}^{I,(2)}-2 \beta_{1} g_{1}^{I,(1)}-6 \zeta_{2} \beta_{0} A_{2}^{I}-3 \zeta_{2} \beta_{1} A_{1}^{I}-2 \beta_{0} C_{I} \overline{\mathcal{G}}_{2}^{(1)}
$$$$
\left.-4 \beta_{0}^{2} C_{I} \overline{\mathcal{G}}_{1}^{(2)}-2 \beta_{1} C_{I} \overline{\mathcal{G}}_{1}^{(1)}\right) \ln \left(\frac{q^{2}}{\mu_{F}^{2}}\right)+\left(5 \beta_{0} \beta_{1} \delta_{I, g}-2 \beta_{0} B_{2}^{I}-\beta_{1} B_{1}^{I}+2 \beta_{0}^{2} g_{1}^{I,(1)}\right.
$$$$
\left.\left.+3 \zeta_{2} \beta_{0}^{2} A_{1}^{I}+2 \beta_{0}^{2} C_{I} \overline{\mathcal{G}}_{1}^{(1)}\right) \ln ^{2}\left(\frac{q^{2}}{\mu_{F}^{2}}\right)+\left(\frac{2}{3} \beta_{0}^{2} B_{1}^{I}-\frac{2}{3} \beta_{0}^{3} \delta_{I, g}\right) \log ^{3}\left(\frac{q^{2}}{\mu_{F}^{2}}\right)\right] \delta(1-z)
$$ 


$$
\begin{aligned}
& +\left[-4 \beta_{0} C_{I} \overline{\mathcal{G}}_{2}^{(1)}-8 \beta_{0}^{2} C_{I} \overline{\mathcal{G}}_{1}^{(2)}-4 \beta_{1} C_{I} \overline{\mathcal{G}}_{1}^{(1)}-2 f_{3}^{I}+\left(4 \beta_{0} f_{2}^{I}+8 \beta_{0}^{2} C_{I} \overline{\mathcal{G}}_{1}^{(1)}\right.\right. \\
& \left.\left.+2 A_{3}^{I}\right) \ln \left(\frac{q^{2}}{\mu_{F}^{2}}\right)+\left(-2 \beta_{0} A_{2}^{I}-\beta_{1} A_{1}^{I}\right) \ln ^{2}\left(\frac{q^{2}}{\mu_{F}^{2}}\right)+\left(\frac{2}{3} \beta_{0}^{2} A_{1}^{I}\right) \ln ^{3}\left(\frac{q^{2}}{\mu_{F}^{2}}\right)\right] \mathcal{D}_{0} \\
& +\left[8 \beta_{0} f_{2}^{I}+16 \beta_{0}^{2} C_{I} \overline{\mathcal{G}}_{1}^{(1)}+4 A_{3}^{I}+\left(-8 \beta_{2} A_{2}^{I}-4 \beta_{1} A_{1}^{I}\right) \ln \left(\frac{q^{2}}{\mu_{F}^{2}}\right)\right. \\
& \left.+\left(4 \beta_{0}^{2} A_{1}^{I}\right) \ln ^{2}\left(\frac{q^{2}}{\mu_{F}^{2}}\right)\right] \mathcal{D}_{1}+\left[-8 \beta_{0} A_{2}^{I}-4 \beta_{1} A_{1}^{I}+\left(8 \beta_{0}^{2} A_{1}^{I}\right) \ln \left(\frac{q^{2}}{\mu_{F}^{2}}\right)\right] \mathcal{D}_{2} \\
& +\left[\left(\frac{16}{3} \beta_{0}^{2} A_{1}^{I}\right)\right] \mathcal{D}_{3}
\end{aligned}
$$

Notice that $\Psi^{I,(1)}$ and $\Psi^{I,(2)}$ are completely known. Using our $\Psi^{I,(1)}$, and $\Psi^{I,(2)}$ we could successfully reproduce soft plus virtual cross section $\Delta_{g}^{s v,(i)}\left(z, q^{2}, \mu_{F}^{2}\right)($ see $[10,11])(i=1,2)$ of the Higgs production from that of DY [8] and vice versa. To compute $\Delta_{I}^{s v,(3)}\left(z, q^{2}, \mu_{F}^{2}\right)$ (equivalently $\Psi^{I,(3)}$ ) we need to know $\overline{\mathcal{G}}_{2}^{I,(2)}$ and $\overline{\mathcal{G}}_{3}^{I,(1)}$ either from DY or Higgs production because these constants are maximally non-abelien. Notice that these constants appear only in the coefficient of $\delta(1-z)$ part of $\Psi^{I,(3)}$. Since the coefficients of $\mathcal{D}_{i}(i=0,1,2,3)$ in $\Psi^{I,(3)}$ do not depend on these unknown constants $\overline{\mathcal{G}}_{3}^{I,(1)}$ and $\overline{\mathcal{G}}_{2}^{I,(2)}$, we can predict these coefficients(say for the Higgs production) by using the universal soft distribution function extracted from a process(say DY), the three loop form factors and the renormalisation constants. Our prediction agrees with the partial $N^{3} L O$ soft plus virtual results [25] for DY and Higgs productions.

With these available informations one can also determine all the quantities upto $N^{3} L L$ level in the threshold resummation. To do this, we first recollect that the soft distribution function is renormalisation group invariant. Its UV divergence can be removed by the coupling constant renormalisation. This introduces a renormalisation scale $\mu_{R}$ which is arbitrary to all orders in perturbation theory. In order to compute various quantities in the threshold resummation formula from the soft distribution function, we choose $\mu_{R}=\mu_{F}$. With this choice, one can express the soft distribution function as a sum of pole and finite parts in $\varepsilon$ as $\varepsilon \rightarrow 0$, that is

$$
\Phi^{I}\left(a_{s}\left(\mu_{F}^{2}\right), \frac{q^{2}}{\mu_{F}^{2}}, z, \varepsilon\right)=\Phi_{\text {pole }}^{I}\left(a_{s}\left(\mu_{F}^{2}\right), \frac{q^{2}}{\mu_{F}^{2}}, z, \frac{1}{\varepsilon}\right)+\Phi_{\text {fin }}^{I}\left(a_{s}\left(\mu_{F}^{2}\right), \frac{q^{2}}{\mu_{F}^{2}}, z, \varepsilon\right)
$$

With this decomposition, it is now straightforward to identify the finite part $\Phi_{\text {fin }}^{I}$ with the threshold resummation formula as

$$
\begin{aligned}
2 \int_{0}^{1} d z z^{N-1} \Phi_{f i n}^{I}\left(a_{s}\left(\mu_{F}^{2}\right), \frac{q^{2}}{\mu_{F}^{2}}, z, \varepsilon=0\right)= & \int_{0}^{1} d z \frac{z^{N-1}-1}{1-z}\left[D^{I}\left(a_{s}\left(q^{2}(1-z)^{2}\right)\right)\right. \\
& \left.+2 \int_{\mu_{F}^{2}}^{q^{2}(1-z)^{2}} \frac{d \lambda^{2}}{\lambda^{2}} A^{I}\left(a_{s}\left(\lambda^{2}\right)\right)\right]
\end{aligned}
$$




$$
+H_{S}^{I}\left(a_{s}\left(\mu_{F}^{2}\right), \frac{q^{2}}{\mu_{F}^{2}}\right)
$$

where the subscript $S$ in $H_{S}^{I}$ indicates that it comes from the soft part of the cross section. The remaining contribution comes from the form factor. $D^{I}\left(a_{s}\left(q^{2}(1-z)^{2}\right)\right)$ can be expanded in powers of bare coupling constant $\hat{a}_{s}$ as follows:

$$
D^{I}\left(a_{s}\left(q^{2}(1-z)^{2}\right)\right)=\sum_{i=1}^{\infty} \hat{a}_{s}^{i}\left(\frac{q^{2}(1-z)^{2}}{\mu^{2}}\right)^{i \frac{\varepsilon}{2}} S_{\varepsilon}^{i} \hat{D}^{I,(i)}(\varepsilon)
$$

The finiteness of $D^{I}$ after coupling constant renormalisation demands that it satisfies the following expansion in $\varepsilon$ :

$$
\hat{D}^{I,(i)}(\varepsilon)=\sum_{j=1-i}^{\infty} \hat{d}_{j}^{I,(i)} \varepsilon^{j}
$$

Using RG invariance, the coefficients of negative powers of $\varepsilon$ can be evaluated as

$$
\begin{aligned}
& \hat{d}_{-1}^{g,(2)}=-2 \beta_{0} \hat{d}_{0}^{g,(1)} \\
& \hat{d}_{-2}^{g,(3)}=4 \beta_{0}^{2} \hat{d}_{0}^{g,(1)} \\
& \hat{d}_{-1}^{g,(3)}=-4 \beta_{0} \hat{d}_{0}^{g,(2)}-4 \beta_{0}^{2} \hat{d}_{1}^{g,(1)}-\beta_{1} \hat{d}_{0}^{g,(1)}
\end{aligned}
$$

We find that for non-negative powers of $\varepsilon(j \geq 0)$,

$$
\begin{aligned}
& \hat{d}_{j}^{I,(1)}=\left.2 \bar{g}_{1}^{I, j+1}\right|_{\mathcal{D}_{0}} \\
& \hat{d}_{j}^{I,(2)}=\left.\left(\bar{g}_{2}^{I, j+1}-2 \beta_{0} \bar{g}_{1}^{I, j+2}\right)\right|_{\mathcal{D}_{0}} \\
& \hat{d}_{j}^{I,(3)}=\left.\left(\frac{2}{3} \bar{g}_{3}^{I, j+1}-\frac{8}{3} \beta_{0} \bar{g}_{2}^{I, j+2}-\frac{2}{3} \beta_{1} \bar{g}_{1}^{I, j+2}+\frac{8}{3} \beta_{0}^{2} \bar{g}_{1}^{I, j+3}\right)\right|_{\mathcal{D}_{0}}
\end{aligned}
$$

Using the above equations, we find explicitly

$$
\begin{aligned}
& \hat{d}_{0}^{g,(1)}=0 \\
& \hat{d}_{1}^{g,(1)}=C_{A}\left(-6 \zeta_{2}\right) \\
& \hat{d}_{2}^{g,(1)}=C_{A}\left(\frac{14}{3} \zeta_{3}\right) \\
& \hat{d}_{0}^{g,(2)}=C_{A}^{2}\left(-\frac{1616}{27}+\frac{308}{3} \zeta_{2}+56 \zeta_{3}\right)+C_{A} n_{f}\left(\frac{224}{27}-\frac{56}{3} \zeta_{2}\right)
\end{aligned}
$$




$$
\begin{aligned}
\hat{d}_{1}^{g,(2)}= & C_{A}^{2}\left(\frac{4856}{81}-\frac{938}{9} \zeta_{2}+8 \zeta_{2}^{2}-\frac{1364}{9} \zeta_{3}\right) \\
& +C_{A} n_{f}\left(-\frac{656}{81}+\frac{140}{9} \zeta_{2}+\frac{248}{9} \zeta_{3}\right) \\
\hat{d}_{0}^{g,(3)}= & C_{A}^{3}\left(-\frac{1235050}{729}-\frac{352}{3} \zeta_{2} \zeta_{3}+\frac{227548}{81} \zeta_{2}-\frac{1584}{5} \zeta_{2}^{2}+\frac{10376^{2}}{3} \zeta_{3}-384 \zeta_{5}\right) \\
& +C_{A}^{2} n_{f}\left(\frac{328388}{729}-\frac{72004}{81} \zeta_{2}+\frac{352}{5} \zeta_{2}^{2}-\frac{26800}{27} \zeta_{3}\right) \\
& +C_{A} C_{F} n_{f}\left(\frac{3422}{27}-44 \zeta_{2}-\frac{64}{5} \zeta_{2}^{2}-\frac{608}{9} \zeta_{3}\right) \\
& +C_{A} n_{f}^{2}\left(-\frac{19456}{729}+\frac{1760}{27} \zeta_{2}+\frac{2080}{27} \zeta_{3}\right)
\end{aligned}
$$

The coefficients $\hat{d}_{j}^{q,(i)}$ for the DY can be obtained using

$$
\hat{d}_{j}^{q,(i)}=\frac{C_{F}}{C_{A}} \hat{d}_{j}^{g,(i)}
$$

because the soft distributions functions are maximally non-abelien. Also, the coefficients of $a_{s}^{i}\left(q^{2}\right) \mathcal{D}_{0}$ in the soft distribution function $\Phi_{\text {fin }}^{I}$ are related to the coefficients $D_{i}^{I}$ that appear in threshold resummation formula. Hence it is straightforward to obtain $D_{i}^{I}$ from the soft distribution function $\Phi_{f i n}^{I}$. We find that $D_{i}^{I}$ are related to $\hat{d}_{k}^{I,(i)}$ and hence $\bar{g}_{i}^{I, k}$ as follows:

$$
\begin{aligned}
D_{1}^{I} & =\hat{d}_{0}^{I,(1)}=\left.2 \bar{g}_{1}^{I, 1}\right|_{\mathcal{D}_{0}} \\
& =2 \overline{\mathcal{G}}_{1}^{I}(\varepsilon=0) \\
D_{2}^{I} & =\hat{d}_{0}^{I,(2)}+2 \beta_{0} \hat{d}_{1}^{I,(1)}=\left.\left(\bar{g}_{2}^{I, 1}+2 \beta_{0} \bar{g}_{1}^{I, 2}\right)\right|_{\mathcal{D}_{0}} \\
& =2 \overline{\mathcal{G}}_{2}^{I}(\varepsilon=0) \\
D_{3}^{I} & =\hat{d}_{0}^{I,(3)}+4 \beta_{0} \hat{d}_{1}^{I,(2)}+\beta_{1} \hat{d}_{1}^{I,(1)}+4 \beta_{0}^{2} \hat{d}_{2}^{I,(1)} \\
& =\left.\left(\frac{2}{3} \bar{g}_{3}^{I, 1}+\frac{4}{3} \beta_{0} \bar{g}_{2}^{I, 2}+\frac{4}{3} \beta_{1} \bar{g}_{1}^{I, 2}+\frac{8}{3} \beta_{0}^{2} \bar{g}_{1}^{I, 3}\right)\right|_{\mathcal{D}_{0}} \\
& =2 \overline{\mathcal{G}}_{3}^{I}(\varepsilon=0)
\end{aligned}
$$


From the available informations on three loop results, we find that the following result holds

$$
D_{i}^{I}=2 \overline{\mathcal{G}}_{i}^{I}(\varepsilon=0) \quad i=1,2,3
$$

The fact that $D_{i}^{I}$ can be expressed entirely in terms of $\bar{G}_{i}^{I}(z, \varepsilon)$ (i.e., in terms of $\bar{g}_{i}^{I, k}$ or $\overline{\mathcal{G}}_{i}^{I}(\varepsilon=0)$ ) which are maximally non-abelien, implies that $D_{i}^{I}$ are also maximally non-abelien. Hence,

$$
D_{i}^{q}=\frac{C_{F}}{C_{A}} D_{i}^{g}
$$

with

$$
\begin{aligned}
D_{1}^{g}= & 0 \\
D_{2}^{g}= & C_{A}^{2}\left(-\frac{1616}{27}+\frac{176}{3} \zeta_{2}+56 \zeta_{3}\right)+C_{A} n_{f}\left(\frac{224}{27}-\frac{32}{3} \zeta_{2}\right) \\
D_{3}^{g}= & C_{A}^{3}\left(-\frac{594058}{729}+\frac{98224}{81} \zeta_{2}+\frac{40144}{27} \zeta_{3}-\frac{2992}{15} \zeta_{2}^{2}-\frac{352}{3} \zeta_{2} \zeta_{3}-384 \zeta_{5}\right) \\
& +C_{A}^{2} n_{f}\left(\frac{125252}{729}-\frac{29392}{81} \zeta_{2}-\frac{2480}{9} \zeta_{3}+\frac{736}{15} \zeta_{2}^{2}\right) \\
& +C_{A} C_{F} n_{f}\left(\frac{3422}{27}-32 \zeta_{2}-\frac{608}{9} \zeta_{3}-\frac{64}{5} \zeta_{2}^{2}\right)+C_{A} n_{f}^{2}\left(-\frac{3712}{729}+\frac{640}{27} \zeta_{2}+\frac{320}{27} \zeta_{3}\right)_{67)}
\end{aligned}
$$

The above results are in agreement with [25-27]. We also find the resummation exponents $D_{i}^{I}$ can be extracted by using the following relations:

$$
\begin{aligned}
& D_{1}^{I}=\left.\Delta_{I}^{s v,(1)}\right|_{\mathcal{D}_{0}} \\
& D_{2}^{I}=\left.\left(\Delta_{I}^{s v,(2)}-\frac{1}{2} \Delta_{I}^{s v,(1)} \otimes \Delta_{I}^{s v,(1)}\right)\right|_{\mathcal{D}_{0}} \\
& D_{3}^{I}=\left.\left(\Delta_{I}^{s v,(3)}-\Delta_{I}^{s v,(1)} \otimes \Delta_{I}^{s v,(2)}+\frac{1}{3} \Delta_{I}^{s v,(1)} \otimes \Delta_{I}^{s v,(1)} \otimes \Delta_{I}^{s v,(1)}\right)\right|_{\mathcal{D}_{0}}
\end{aligned}
$$

where $\Delta_{I}^{s v,(i)}$ in the above are computed at the scale $\mu_{F}^{2}=\mu_{R}^{2}=q^{2}$. From the following convolution identity [37] upto irrelevant regular terms(denoted by $\cdots$...

$$
\mathcal{D}_{i} \otimes \mathcal{D}_{j}=d_{i j} \delta(1-z)+\sum_{l=0}^{i+j+1} c_{i j, l} \mathcal{D}_{l}+\cdots
$$

it is interesting to notice that in order to obtain $D_{i}^{I}$, it is sufficient to know the coefficients of all $\mathcal{D}_{l}$ $\left(l=l_{\text {max }}\right.$ to 0 ) (that means, we need not know the information on the coefficient of $\delta(1-z)$ function 
and the regular part of $\Delta_{I}^{s v,(i)}$ ) and the complete soft information of $\Delta_{I}^{s v,(i-1)}$ (i.e., the coefficients of all $\mathcal{D}_{i}$ and $\delta(1-z)$ are needed).

Finally, the coefficient of $\delta(1-z)$ in the resummation formula can be obtained from $\Phi_{\text {fin }}^{I}$ by defining the coupling constant at the scale $\mu_{F}^{2}$. The result is

$$
H_{S}^{I}\left(a_{s}\left(\mu_{F}^{2}\right), \frac{q^{2}}{\mu_{F}^{2}}\right)=\sum_{i=1}^{\infty} a_{S}^{i}\left(\mu_{F}^{2}\right) H_{S, i}^{I}
$$

where

$$
\begin{aligned}
H_{S, 1}^{g}= & -3 \zeta_{2}+\ln ^{2}\left(\frac{q^{2}}{\mu_{F}^{2}}\right) \\
H_{S, 2}^{g}= & C_{A}^{2}\left(-\frac{164}{81}+\frac{35}{9} \zeta_{2}+\frac{34}{9} \zeta_{3}+\left(-\frac{8}{3} \zeta_{2}+\frac{56}{27}\right) \ln \left(\frac{q^{2}}{\mu_{F}^{2}}\right)\right. \\
& \left.-\frac{10}{9} \ln ^{2}\left(\frac{q^{2}}{\mu_{F}^{2}}\right)+\frac{2}{9} \ln ^{3}\left(\frac{q^{2}}{\mu_{F}^{2}}\right)\right)+C_{A} n_{f}\left(\frac{1214}{81}-\frac{469}{18} \zeta_{2}+2 \zeta_{2}^{2}-\frac{187}{9} \zeta_{3}\right. \\
& +\left(\frac{44}{3} \zeta_{2}+14 \zeta_{3}-\frac{404}{27}\right) \ln \left(\frac{q^{2}}{\mu_{F}^{2}}\right)+\left(-2 \zeta_{2}+\frac{67}{9}\right) \ln ^{2}\left(\frac{q^{2}}{\mu_{F}^{2}}\right) \\
& \left.-\frac{11}{9} \ln ^{3}\left(\frac{q^{2}}{\mu_{F}^{2}}\right)\right)
\end{aligned}
$$

and

$$
H_{S}^{q,(i)}=\frac{C_{F}}{C_{A}} H_{S}^{g,(i)}
$$

The remaining contribution to the exponent comes from the the finite part of form factor.

We conclude our discussion on this subject with a brief discussion on the corresponding soft as well as jet distribution functions that appear in deep inelastic scattering. The soft plus virtual coefficient function $c_{I, 2}^{s v}\left(Q^{2}, z\right)$ that appear in the hadronic structure function $F_{2}$ can be expressed as

$$
\begin{aligned}
C \ln c_{I, 2}^{s v}\left(Q^{2}, z\right)= & \left(\ln \left(Z^{I}\left(\hat{a}_{s}, \mu_{R}^{2}, \mu^{2}, \varepsilon\right)\right)^{2}+\ln \left|\hat{F}^{I}\left(\hat{a}_{s}, Q^{2}, \mu^{2}, \varepsilon\right)\right|^{2}\right) \delta(1-z) \\
& +2 \Phi_{S J}^{I}\left(\hat{a}_{s}, Q^{2}, \mu^{2}, z, \varepsilon\right)-C \ln \Gamma_{I I}\left(\hat{a}_{s}, \mu^{2}, \mu_{F}^{2}, z, \varepsilon\right)
\end{aligned}
$$

where $\Phi_{S J}^{I}\left(\hat{a}_{s}, Q^{2}, \mu^{2}, z, \varepsilon\right)$ is sum of soft and jet distribution functions. We find that this soft plus jet distribution function also satisfies Sudakov type integro differential equation (see eqn. (35) which can be solved in the same way we solved soft distribution functions. We find that this soft plus jet 
distribution function $\Phi_{S J}^{I}$ can be expressed as

$$
\begin{aligned}
\Phi_{S J}^{I}\left(\hat{a}_{s}, Q^{2}, \mu^{2}, z, \varepsilon\right) & =\Phi_{S J}^{I}\left(\hat{a}_{s}, Q^{2}(1-z), \mu^{2}, \varepsilon\right) \\
& =\sum_{i=1}^{\infty} \hat{a}_{s}^{i}\left(\frac{Q^{2}(1-z)}{\mu^{2}}\right)^{i \frac{\varepsilon}{2}} S_{\varepsilon}^{i}\left(\frac{i \varepsilon}{2(1-z)}\right) \hat{\xi}^{I,(i)}(\varepsilon)
\end{aligned}
$$

where

$$
\hat{\xi}^{I,(i)}(\varepsilon)=\hat{\mathcal{L}}_{F}^{I,(i)}(\varepsilon)\left(A^{I} \rightarrow-A^{I}, G^{I}(\varepsilon) \rightarrow \widetilde{\mathcal{G}}^{I}(\varepsilon)\right)
$$

We find that the constants $\widetilde{\mathcal{G}}^{I}(\varepsilon)$ have the following expansion in terms of $B_{i}^{I}, f_{i}^{I}$ and the $\varepsilon$ dependent part of lower order coefficient functions.

$$
\begin{aligned}
& \widetilde{\mathcal{G}}_{1}^{q}(\varepsilon)=-\left(B_{1}^{q}+f_{1}^{q}\right)+\sum_{k=1}^{\infty} \varepsilon^{k} \widetilde{\mathcal{G}}_{1}^{q,(k)} \\
& \widetilde{\mathcal{G}}_{2}^{q}(\varepsilon)=-\left(B_{2}^{q}+f_{2}^{q}\right)-2 \beta_{0} \widetilde{\mathcal{G}}_{1}^{q,(1)}+\sum_{k=1}^{\infty} \varepsilon^{k} \widetilde{\mathcal{G}}_{2}^{q,(k)} \\
& \widetilde{\mathcal{G}}_{3}^{q}(\varepsilon)=-\left(B_{3}^{q}+f_{3}^{q}\right)-2 \beta_{1} \widetilde{\mathcal{G}}_{1}^{q,(1)}-2 \beta_{0}\left(\widetilde{\mathcal{G}}_{2}^{q,(1)}+2 \beta_{0} \widetilde{\mathcal{G}}_{1}^{q,(2)}\right)+\sum_{k=1}^{\infty} \varepsilon^{k} \widetilde{\mathcal{G}}_{3}^{q,(k)}
\end{aligned}
$$

The $z$ independent constants $\widetilde{\mathcal{G}}_{i}^{q,(k)}$ are computed using the coefficient functions $c_{q, 2}^{s v}\left(z, Q^{2}\right)$ known upto three loop level $[23,38]$. Recollect that the three loop form factors were obtained from these coefficient functions by demanding the finiteness of the partonic cross sections after mass factorisation and also notice that the method used there is very different from the method presented in this paper. We obtain

$$
\begin{aligned}
\widetilde{\mathcal{G}}_{1}^{q,(1)}= & C_{F}\left(\frac{7}{2}-3 \zeta_{2}\right) \\
\widetilde{\mathcal{G}}_{1}^{q,(2)}= & C_{F}\left(-\frac{7}{2}+\frac{9}{8} \zeta_{2}+\frac{7}{3} \zeta_{3}\right) \\
\widetilde{\mathcal{G}}_{2}^{q,(1)}= & C_{F}^{2}\left(\frac{9}{8}-\frac{41}{2} \zeta_{2}+\frac{82}{5} \zeta_{2}^{2}-6 \zeta_{3}\right) \\
& +C_{F} C_{A}\left(\frac{69761}{648}-\frac{1961}{36} \zeta_{2}-\frac{17}{5} \zeta_{2}^{2}-40 \zeta_{3}\right) \\
& +C_{F} n_{f}\left(-\frac{5569}{324}+\frac{163}{18} \zeta_{2}+4 \zeta_{3}\right)
\end{aligned}
$$

Using the following decomposition,

$$
\Phi_{S J}^{I}\left(a_{S}\left(\mu_{F}^{2}\right), \frac{Q^{2}}{\mu_{F}^{2}}, z, \varepsilon\right)=\Phi_{S J, p o l e}^{I}\left(a_{S}\left(\mu_{F}^{2}\right), \frac{Q^{2}}{\mu_{F}^{2}}, z, \frac{1}{\varepsilon}\right)+\Phi_{S J, f i n}^{I}\left(a_{S}\left(\mu_{F}^{2}\right), \frac{Q^{2}}{\mu_{F}^{2}}, z, \varepsilon\right)
$$


it is now straightforward to identify the finite part $\Phi_{S J, f i n}^{I}$ with the DIS threshold resummation formula as

$$
\begin{aligned}
2 \int_{0}^{1} d z z^{N-1} \Phi_{S J, f i n}^{I}\left(a_{S}\left(\mu_{F}^{2}\right), \frac{Q^{2}}{\mu_{F}^{2}}, z, \varepsilon=0\right)= & \int_{0}^{1} d z \frac{z^{N-1}-1}{1-z}\left[B_{D I S}^{I}\left(a_{S}\left(Q^{2}(1-z)\right)\right)\right. \\
& \left.+\int_{\mu_{F}^{2}}^{Q^{2}(1-z)} \frac{d \lambda^{2}}{\lambda^{2}} A^{I}\left(a_{S}\left(\lambda^{2}\right)\right)\right] \\
& +H_{S J, S}^{I}\left(a_{S}\left(\mu_{F}^{2}\right), \frac{Q^{2}}{\mu_{F}^{2}}\right)
\end{aligned}
$$

Using the above equation, we find that the resummation constants $B_{D I S, i}^{q}$ satisfy the following relation

$$
B_{D I S, i}^{q}=\widetilde{\mathcal{G}}_{i}^{q}(\varepsilon=0) \quad i=1,2,3
$$

The resulting $B_{D I S, i}^{q}$ s agree with those given in [23].

To summarise, we have extracted the soft distribution function $\Phi^{I}$ using mass factorisation formula for both Drell-Yan as well as Higgs productions within the framework of perturbative QCD. This is possible now thanks to various three loop results available for the form factors and splitting functions. The $\Phi^{I}$ is known completely upto two loop level. Except the soft bremsstrahlung contributions proportional to $\delta(1-z)$ (at three loop level), all the other soft terms $\left(\mathcal{D}_{i}\right)$ are known for the soft distribution functions $\Phi^{I}$ upto three loop level. We have also shown that the soft distribution functions satisfy Sudakov type integro-differential equation that the quark and gluon form factors satisfy. We found that they are process independent. In other words, knowing the soft distribution function of the Drell-Yan process, one can obtain the same for the Higgs production by simply multiplying the colour factor combination $C_{A} / C_{F}$. Hence, unlike the cross sections $\Delta_{I}^{s v}$, these soft distribution functions are maximally non-abelien. Notice that the form factors have this property only at the pole level. Using the universal soft distribution function extracted from DY production, we could reproduce the soft plus virtual cross section for the Higgs production using the gluon form factor and its renormalisation constant. We have also shown how these soft distribution functions are related to threshold resummation formula. We have extracted various coefficients appearing in threshold resummation formula upto three loop level using the soft distribution function. We have also discussed the DIS soft and jet distribution functions in the present context.

Acknowledgments: The author would like to thank S. Moch and A. Vogt for providing the constants $S_{i}$ of DY that are computed in their paper [25].

\section{References}

[1] A. Djouadi, arXiv:hep-ph/0503172. 
[2] A. Djouadi, arXiv:hep-ph/0503173.

[3] G. Altarelli, R. K. Ellis and G. Martinelli, Nucl. Phys. B 143, 521 (1978) [Erratum-ibid. B 146, 544 (1978)].

[4] S. Dawson, Nucl. Phys. B 359, 283 (1991).

[5] A. Djouadi, M. Spira and P. M. Zerwas, Phys. Lett. B 264, 440 (1991).

[6] M. Spira, A. Djouadi, D. Graudenz and P. M. Zerwas, Nucl. Phys. B 453, 17 (1995) [arXiv:hep-ph/9504378].

[7] T. Matsuura and W. L. van Neerven, Z. Phys. C 38, 623 (1988).

[8] T. Matsuura, S. C. van der Marck and W. L. van Neerven, Nucl. Phys. B 319, 570 (1989).

[9] R. Hamberg, W. L. van Neerven and T. Matsuura, Nucl. Phys. B 359, 343 (1991) [Erratumibid. B 644, 403 (2002)].

[10] R. V. Harlander and W. B. Kilgore, Phys. Rev. D 64, 013015 (2001) [arXiv:hep-ph/0102241].

[11] S. Catani, D. de Florian and M. Grazzini, JHEP 0105, 025 (2001) [arXiv:hep-ph/0102227].

[12] R. V. Harlander and W. B. Kilgore, Phys. Rev. Lett. 88, 201801 (2002) [arXiv:hep-ph/0201206].

[13] C. Anastasiou and K. Melnikov, Nucl. Phys. B 646, 220 (2002) [arXiv:hep-ph/0207004].

[14] V. Ravindran, J. Smith and W. L. van Neerven, Nucl. Phys. B 665, 325 (2003) [arXiv:hep-ph/0302135].

[15] G. Sterman, Nucl. Phys. B 281, 310 (1987).

[16] S. Catani and L. Trentadue, Nucl. Phys. B 327, 323 (1989).

[17] A. Vogt, Phys. Lett. B 497, 228 (2001) [arXiv:hep-ph/0010146].

[18] S. Catani, D. de Florian, M. Grazzini and P. Nason, JHEP 0307, 028 (2003) [arXiv:hep-ph/0306211].

[19] S. Moch, J. A. M. Vermaseren and A. Vogt, Nucl. Phys. B 688, 101 (2004) [arXiv:hep-ph/0403192].

[20] A. Vogt, S. Moch and J. A. M. Vermaseren, Nucl. Phys. B 691, 129 (2004) [arXiv:hep-ph/0404111].

[21] S. Moch, J. A. M. Vermaseren and A. Vogt, JHEP 0508, 049 (2005) arXiv:hep-ph/0507039.

[22] S. Moch, J. A. M. Vermaseren and A. Vogt, Phys. Lett. B 625, 245 (2005) [arXiv:hep-ph/0508055]. 
[23] S. Moch, J. A. M. Vermaseren and A. Vogt, Nucl. Phys. B 726, 317 (2005) [arXiv:hep-ph/0506288].

[24] J. Blumlein and J. A. M. Vermaseren, Phys. Lett. B 606, 130 (2005) arXiv:hep-ph/0411111].

[25] S. Moch and A. Vogt, Phys. Lett. B 631, 48 (2005) [arXiv:hep-ph/0508265].

[26] E. Laenen and L. Magnea, arXiv:hep-ph/0508284.

[27] A. Idilbi, X. d. Ji, J. P. Ma and F. Yuan, arXiv:hep-ph/0509294.

[28] K. G. Chetyrkin, B. A. Kniehl and M. Steinhauser, Nucl. Phys. B 510, 61 (1998) [arXiv:hep-ph/9708255].

[29] V. V. Sudakov, Sov. Phys. JETP 3, 65 (1956) [Zh. Eksp. Teor. Fiz. 30, 87 (1956)].

[30] A. H. Mueller, Phys. Rev. D 20, 2037 (1979).

[31] J. C. Collins, Phys. Rev. D 22, 1478 (1980).

[32] A. Sen, Phys. Rev. D 24, 3281 (1981).

[33] V. Ravindran, J. Smith and W. L. van Neerven, Nucl. Phys. B 704, 332 (2005) [arXiv:hep-ph/0408315].

[34] J. Blumlein, V. Ravindran and W. L. van Neerven, Nucl. Phys. B 586, 349 (2000) [arXiv:hep-ph/0004172].

[35] J. Blumlein and V. Ravindran, Nucl. Phys. B 716, 128 (2005) arXiv:hep-ph/0501178].

[36] Y. L. Dokshitzer, G. Marchesini and G. P. Salam, arXiv:hep-ph/0511302.

[37] W. L. van Neerven and A. Vogt, Nucl. Phys. B 603, 42 (2001) [arXiv:hep-ph/0103123.

[38] W. L. van Neerven and E. B. Zijlstra, Phys. Lett. B 272, 127 (1991). 